Acta Crystallographica Section C

Crystal Structure

Communications

ISSN 0108-2701

Editor: George Ferguson

\title{
3-(o-Carboxyphenyl)-1-methyltriazene 1-Oxide
}

\author{
C. Samanta, S. C. Saha and A. K. Mukherjee
}

This electronic document was scanned from an archival copy of material deposited to accompany a paper published in an IUCr journal. In many cases the only accessible copy was a microfilm of a poor-quality original. 
Table . Observed and calculated structure factors for triazene

Page 1

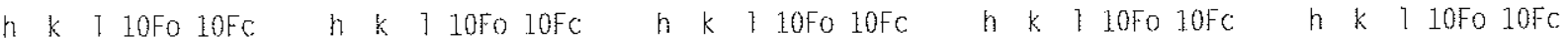

$\begin{array}{rrrrr}2 & 0 & 0 & 255 & 126 \\ 4 & 0 & 0 & 102 & 288 \\ 6 & 0 & 0 & 210 & 2 \\ 8 & 0 & 0 & 86 & 66 \\ 10 & 0 & 0 & 174 & 126 \\ 12 & 0 & 0 & 84 & 24 \\ 14 & 0 & 0 & 115 & 129 \\ 16 & 0 & 0 & 77 & 35 \\ 18 & 0 & 0 & 115 & 69 \\ 1 & 1 & 0 & 78 & 55 \\ 3 & 1 & 0 & 130 & 390 \\ 5 & 1 & 0 & 58 & 46 \\ 7 & 1 & 0 & 110 & 69 \\ 9 & 1 & 0 & 109 & 97\end{array}$

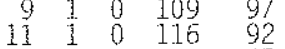

$\begin{array}{rrrrr}13 & 1 & 0 & 143 & 25 \\ 15 & 1 & 0 & 91 & 54\end{array}$

$\begin{array}{lllll}17 & 1 & 0 & 44 & 26 \\ 0 & 2 & 0 & 99 & 684\end{array}$

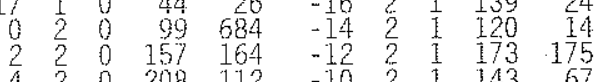

$\begin{array}{lllll}4 & 2 & 0 & 208 & 112 \\ 6 & 2 & 0 & 224 & 225 \\ 0 & 2 & 0 & 227 & 57\end{array}$

$\begin{array}{llllll}10 & 2 & 0 & 147 & 73\end{array}$

$\begin{array}{rrrrr}12 & 2 & 0 & 109 & 167 \\ 14 & 2 & 0 & 48 & 24\end{array}$

$\begin{array}{rrrrr}14 & 2 & 0 & 48 & 24 \\ 16 & 2 & 0 & 44 & 6\end{array}$

$\begin{array}{lllll}18 & 2 & 0 & 108 & 53\end{array}$

$\begin{array}{rrrrr}1 & 3 & 0 & 39 & 242 \\ 3 & 3 & 0 & 101 & 11\end{array}$

$\begin{array}{rrrrr}5 & 3 & 0 & 190 & 91 \\ 7 & 3 & 0 & 207 & 137\end{array}$

$\begin{array}{rrrrr}9 & 3 & 0 & 149 & 110 \\ 1 & 3 & 0 & 165 & 85\end{array}$

$\begin{array}{rrrrr}13 & 3 & 0 & 151 & 148 \\ 15 & 3 & 0 & 157 & 60\end{array}$

$\begin{array}{rrrrr}15 & 3 & 0 & 157 & 00 \\ 17 & 3 & 0 & 161 & 60\end{array}$

$\begin{array}{rrrrr}0 & 4 & 0 & 123 & 184 \\ 2 & 4 & 0 & 98 & 155\end{array}$

$\begin{array}{rrrrr}4 & 4 & 0 & 29 & 122 \\ 6 & 4 & 0 & 140 & 153\end{array}$

$\begin{array}{lllll}6 & 4 & 0 & 140 & 123 \\ 8 & 4 & 0 & 104 & 120\end{array}$

$\begin{array}{rrrrr}10 & 4 & 0 & 44 & 58 \\ 12 & 4 & 0 & 87 & 14\end{array}$

$\begin{array}{lllll}14 & 4 & 0 & 56 & 14\end{array}$

$\begin{array}{rrrrrrrrrr}16 & 4 & 0 & 60 & 50 & 3 & 3 & 1 & 93 & 197 \\ 18 & 4 & 0 & 37 & 19 & 5 & 3 & 1 & 161 & 57 \\ 1 & 5 & 0 & 89 & 91 & 7 & 3 & 1 & 238 & 86 \\ 3 & 5 & 0 & 79 & 77 & 9 & 3 & 1 & 159 & 57\end{array}$

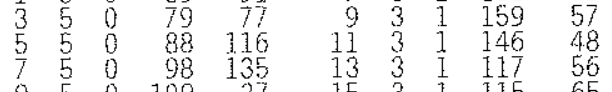

$\begin{array}{rrrrr}9 & 5 & 0 & 109 & 37 \\ 11 & 5 & 0 & 112 & 85\end{array}$

$\begin{array}{rrrrr}11 & 5 & 0 & 112 & 85 \\ 13 & 5 & 0 & 89 & 125\end{array}$

$\begin{array}{lllll}15 & 5 & 0 & 111 & 81 \\ 17 & 5 & 0 & 140 & 110\end{array}$

$\begin{array}{rrrrr}0 & 6 & 0 & 65 & 83 \\ 2 & 6 & 0 & 101 & 47\end{array}$

$\begin{array}{rrrrr}3 & 6 & 0 & 101 & 47 \\ 4 & 6 & 0 & 94 & 159 \\ 6 & 6 & 0 & 68 & 87\end{array}$

$\begin{array}{rrrrrrrrrr}6 & 6 & 0 & 68 & 87 & -6 & 4 & 1 & 168 & 46 \\ 8 & 6 & 0 & 39 & 168 & -4 & 4 & 1 & 163 & 7 \\ 10 & 6 & 0 & 37 & 22 & -2 & 4 & 1 & 124 & 230\end{array}$

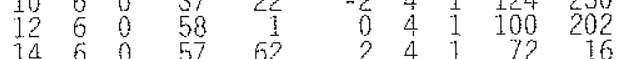

$\begin{array}{rrrrrrrrrr}14 & 6 & 0 & 57 & 62 & 2 & 4 & 1 & 72 & 16 \\ 16 & 6 & 0 & 75 & 61 & 4 & 4 & 1 & 69 & 21 \\ 18 & 6 & 0 & 41 & 50 & 6 & 4 & 1 & 74 & 55\end{array}$

$\begin{array}{rrrrrrrrrr}18 & 6 & 0 & 41 & 50 & 6 & 4 & 1 & 74 & 55 \\ 1 & 7 & 0 & 60 & 102 & 8 & 4 & 1 & 29 & 91 \\ 3 & 7 & 0 & 0 & 48 & 10 & 4 & 1 & 115 & 102\end{array}$

$\begin{array}{rrrrrrrrrr}5 & 7 & 0 & 77 & 59 & 12 & 4 & 1 & 117 & 50 \\ 7 & 7 & 0 & 80 & 125 & 14 & 4 & 1 & 130 & 80 \\ 9 & 7 & 0 & 111 & 67 & 16 & 4 & 1 & 141 & 43\end{array}$

$\begin{array}{rrrrrrrrrr}9 & 7 & 0 & 111 & 67 & 16 & 4 & 1 & 141 & 43 \\ 11 & 7 & 0 & 116 & 37 & 18 & 4 & 1 & 141 & 16 \\ 13 & 7 & 0 & 111 & 17 & -17 & 5 & 1 & 62 & 93 \\ 15 & 7 & 0 & 112 & 130 & -15 & 5 & 1 & 73 & 103\end{array}$

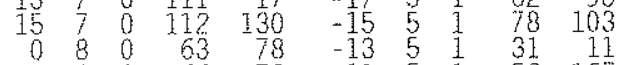

$\begin{array}{llllllllll}2 & 8 & 0 & 63 & 78 & -13 & 5 & 1 & 31 & 11 \\ 4 & 8 & 0 & 66 & 78 & -11 & 5 & 1 & 52 & 167 \\ 4 & 8 & 0 & 82 & 3 & -9 & 5 & 1 & 30 & 164\end{array}$

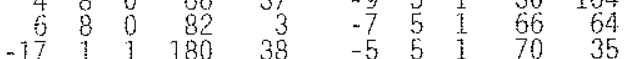

$\begin{array}{lllll}-17 & 1 & 1 & 180 & 38 \\ -15 & 1 & 1 & 145 & 85\end{array}$

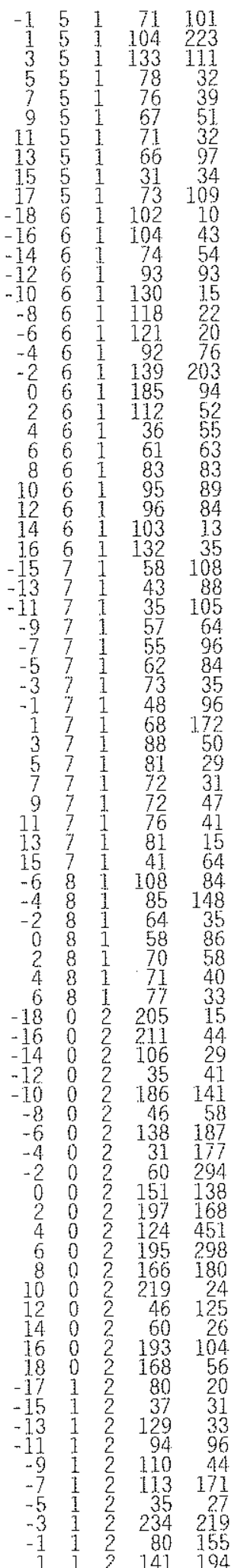

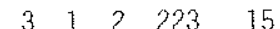

$\begin{array}{rrrrr}15 & 5 & 2 & 65 & 48 \\ 17 & 5 & 2 & 100 & 149\end{array}$

$\begin{array}{rrrrr}-18 & 6 & 2 & 56 & 14 \\ -16 & 6 & 2 & 27 & 57\end{array}$

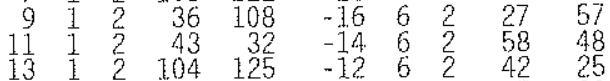

$\begin{array}{rrrrrrrrrr}13 & 1 & 2 & 104 & 125 & -12 & 6 & 2 & 42 & 25 \\ 15 & 1 & 2 & 112 & 74 & -10 & 6 & 2 & 37 & 28 \\ 17 & 1 & 2 & 30 & 56 & -8 & 6 & 2 & 0 & 30\end{array}$

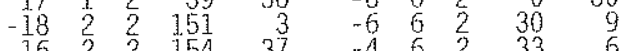

$\begin{array}{llllllllll}-16 & 2 & 2 & 154 & 37 & -4 & 6 & 2 & 33 & 6 \\ -14 & 2 & 2 & 157 & 81 & -2 & 6 & 2 & 120 & 120\end{array}$

$\begin{array}{rrrrrrrrrr}-12 & 2 & 2 & 164 & 40 & 0 & 6 & 2 & 49 & 44 \\ -10 & 2 & 2 & 152 & 28 & 2 & 6 & 2 & 134 & 38\end{array}$

$\begin{array}{rrrrrrrrrr}-8 & 2 & 2 & 170 & 83 & 4 & 6 & 2 & 54 & 81\end{array}$

$\begin{array}{rrrrr}-6 & 2 & 2 & 153 & 200 \\ -4 & 2 & 2 & 77 & 138\end{array}$

$\begin{array}{rrrrr}-2 & 2 & 2 & 117 & 83 \\ 0 & 2 & 2 & 48 & 578\end{array}$

$\begin{array}{lllll}2 & 2 & 2 & 154 & 101\end{array}$

$\begin{array}{rrrrr}4 & 2 & 2 & 160 & 63 \\ 6 & 2 & 2 & 134 & 213 \\ 8 & 2 & 2 & 200 & 57\end{array}$

$\begin{array}{rrrrr}8 & 2 & 2 & 200 & 57 \\ 10 & 2 & 2 & 184 & 108\end{array}$

$\begin{array}{llllr}12 & 2 & 2 & 184 & 4.1\end{array}$

$\begin{array}{lllll}14 & 2 & 2 & 168 & 59 \\ 16 & 2 & 2 & 145 & 8\end{array}$

$\begin{array}{rrrrr}16 & 2 & 2 & 145 & 8 \\ 18 & 2 & 2 & 137 & 37\end{array}$

$\begin{array}{rrrrr}-17 & 3 & 2 & 87 & 46 \\ -15 & 3 & 2 & 90 & 117\end{array}$

$\begin{array}{rrrrr}13 & 3 & 2 & 115 & 69 \\ -1 & 3 & 2 & 125 & 57\end{array}$

$\begin{array}{lllll}-9 & 3 & 2 & 165 & 122\end{array}$

$\begin{array}{rrrrr}-7 & 3 & 2 & 234 & 36\end{array}$

$\begin{array}{rrrrr}-5 & 3 & 2 & 191 & 41 \\ -3 & 3 & 2 & 122 & 204\end{array}$

$\begin{array}{lllll}-1 & 3 & 2 & 141 & 75\end{array}$

$\begin{array}{rrrrr}-1 & 3 & 2 & 141 & 75 \\ 1 & 3 & 2 & 130 & 70\end{array}$

$\begin{array}{rrrrr}3 & 3 & 2 & 56 & 85 \\ 5 & 3 & 2 & 149 & 156\end{array}$

$\begin{array}{rrrrr}7 & 3 & 2 & 190 & 27\end{array}$

$\begin{array}{rrrrr}9 & 3 & 2 & 111 & 59 \\ 11 & 3 & 2 & 83 & 5\end{array}$

$\begin{array}{rrrrr}11 & 3 & 2 & 83 & 5 \\ 13 & 3 & 2 & 35 & 100 \\ 15 & 3 & 2 & 107 & 4\end{array}$

$\begin{array}{lllll}6 & 6 & 2 & 45 & 19 \\ 8 & 6 & 2 & 69 & 36\end{array}$

$\begin{array}{lllll}10 & 6 & 2 & 56 & 72 \\ 12 & 6 & 2 & 34 & 51\end{array}$

$\begin{array}{rrrrr}14 & 6 & 2 & 0 & 75 \\ 16 & 6 & 2 & 42 & 97\end{array}$

$\begin{array}{rrrrr}-15 & 7 & 2 & 37 & 113\end{array}$

$\begin{array}{rrrrr}-13 & 7 & 2 & 72 & 190 \\ -11 & 7 & 2 & 113 & 12\end{array}$

$\begin{array}{rrrrr}-9 & 7 & 2 & 128 & 160 \\ -7 & 7 & 2 & 132 & 79\end{array}$

$\begin{array}{lllll}-5 & 7 & 2 & 109 & 67\end{array}$

$\begin{array}{lllll}-3 & 7 & 2 & 78 & 141\end{array}$

$\begin{array}{rrrrr}-1 & 7 & 2 & 112 & 52 \\ 1 & 7 & 2 & 127 & 40\end{array}$

$\begin{array}{rrrrr}3 & 7 & 2 & 93 & 36 \\ 5 & 7 & 2 & 79 & 68\end{array}$

$\begin{array}{lllll}7 & 7 & 2 & 90 & 88\end{array}$

$\begin{array}{rrrrr}9 & 7 & 2 & 85 & 125 \\ 11 & 7 & 2 & 71 & 227\end{array}$

$\begin{array}{lllll}13 & 7 & 2 & 57 & 229 \\ 15 & 7 & 2 & 78 & 202\end{array}$

$\begin{array}{rrrrr}15 & 7 & 2 & 78 & 202 \\ -6 & 8 & 2 & 68 & 55\end{array}$

$\begin{array}{lllll}-4 & 8 & 2 & 62 & 48 \\ -2 & 8 & 2 & 56 & 66\end{array}$

$\begin{array}{lllll}0 & 8 & 2 & 20 & 20\end{array}$

$\begin{array}{rrrrr}2 & 8 & 2 & 70 & 25 \\ 4 & 8 & 2 & 93 & 71\end{array}$

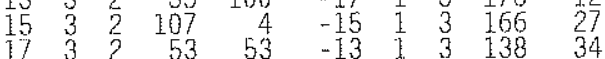

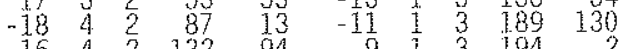

$\begin{array}{llllllllll}-16 & 4 & 2 & 132 & 94 & -9 & 1 & 3 & 194 & 2\end{array}$

$\begin{array}{rrrrr}-14 & 4 & 2 & 77 & 59 \\ -12 & 4 & 2 & 97 & 136\end{array}$

$\begin{array}{rrrrr}-10 & 4 & 2 & 28 & 12 \\ -8 & 4 & 2 & 55 & 44\end{array}$

$\begin{array}{lllll}-8 & 4 & 2 & 55 & 44 \\ -6 & 4 & 2 & 24 & 35\end{array}$

$\begin{array}{llllr}-4 & 4 & 2 & 80 & 26\end{array}$

$\begin{array}{rrrrr}-2 & 4 & 2 & 34 & 737 \\ 0 & 4 & 2 & 61 & 235\end{array}$

$\begin{array}{lllll}0 & 4 & 2 & 61 & 235 \\ 2 & 4 & 2 & 55 & 179\end{array}$

$\begin{array}{rrrrr}4 & 4 & 2 & 60 & 27 \\ 6 & 4 & 2 & 134 & 127\end{array}$

$\begin{array}{lllll}8 & 4 & 2 & 134 & 263\end{array}$

$\begin{array}{rrrrr}8 & 4 & 2 & 134 & 263 \\ 10 & 4 & 2 & 87 & 34\end{array}$

$\begin{array}{lllll}12 & 4 & 2 & 97 & 11 \\ 14 & 4 & 2 & 64 & 55\end{array}$

$\begin{array}{lllll}14 & 4 & 2 & 64 & 55\end{array}$

$\begin{array}{rrrrr}18 & 4 & 2 & 101 & 74 \\ -17 & 5 & 2 & 33 & 23\end{array}$

$\begin{array}{rrrrr}-5 & 1 & 3 & 152 & 289 \\ -3 & 1 & 3 & 171 & 78\end{array}$

$\begin{array}{lllll}-1 & 1 & 3 & 180 & 636 \\ 1 & 1 & 3 & 137 & 167\end{array}$

$\begin{array}{lllll}3 & 1 & 3 & 215 & 107\end{array}$

$\begin{array}{lllll}5 & 1 & 3 & 128 & 239\end{array}$

$\begin{array}{lllll}9 & 1 & 3 & 150 & 126\end{array}$

$\begin{array}{llllr}11 & 1 & 3 & 132 & 29\end{array}$

$\begin{array}{lllll}13 & 1 & 3 & 155 & 66 \\ 15 & 1 & 3 & 151 & 42\end{array}$

$\begin{array}{lllll}17 & 1 & 3 & 163 & 48\end{array}$

$\begin{array}{lllll}-18 & 2 & 3 & 135 & 34 \\ -16 & 2 & 3 & 101 & 56\end{array}$

$\begin{array}{rrrrr}-14 & 2 & 3 & 13 & 159\end{array}$

$\begin{array}{lllll}-12 & 2 & 3 & 71 & 45\end{array}$

$\begin{array}{rrrrrrrrrr}-17 & 5 & 2 & 33 & 23 & -10 & 2 & 3 & 118 & 95 \\ -15 & 5 & 2 & 80 & 175 & -8 & 2 & 3 & 165 & 74 \\ -13 & 5 & 2 & 103 & 118 & -6 & 2 & 3 & 185 & 68 \\ -11 & 5 & 2 & 106 & 71 & -1 & 2 & 3 & 113 & 69\end{array}$

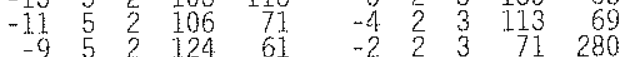

$\begin{array}{llllllllll}-7 & 5 & 2 & 117 & 18 & 0 & 2 & 3 & 56 & 158\end{array}$

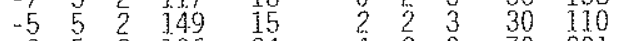

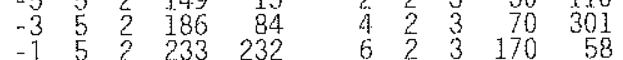

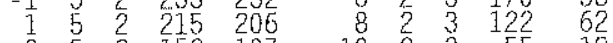

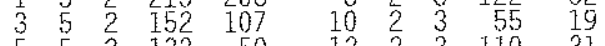

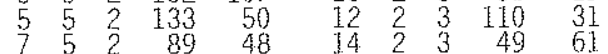

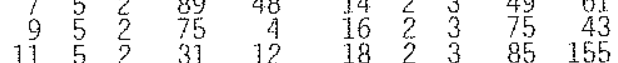

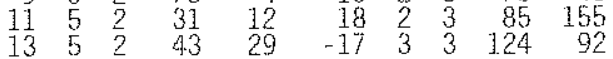
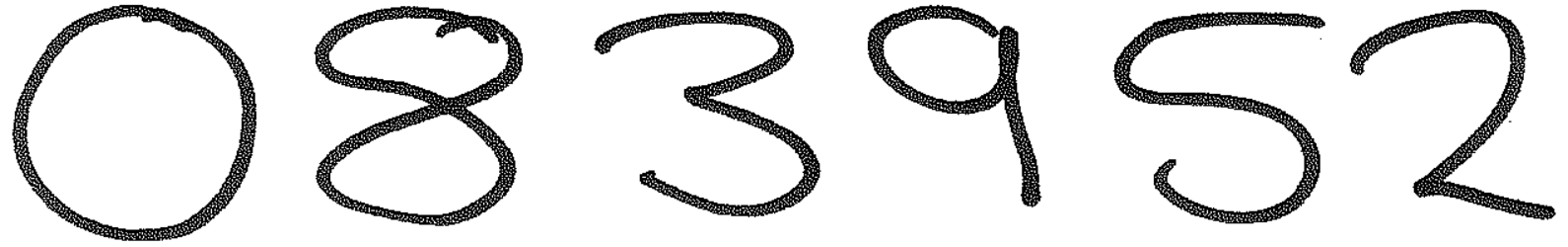
Table . Observed and calculated structure factors for triazene

Page 2

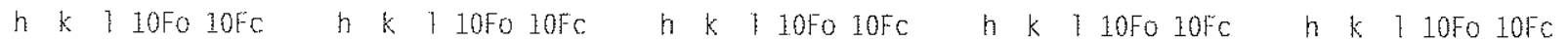

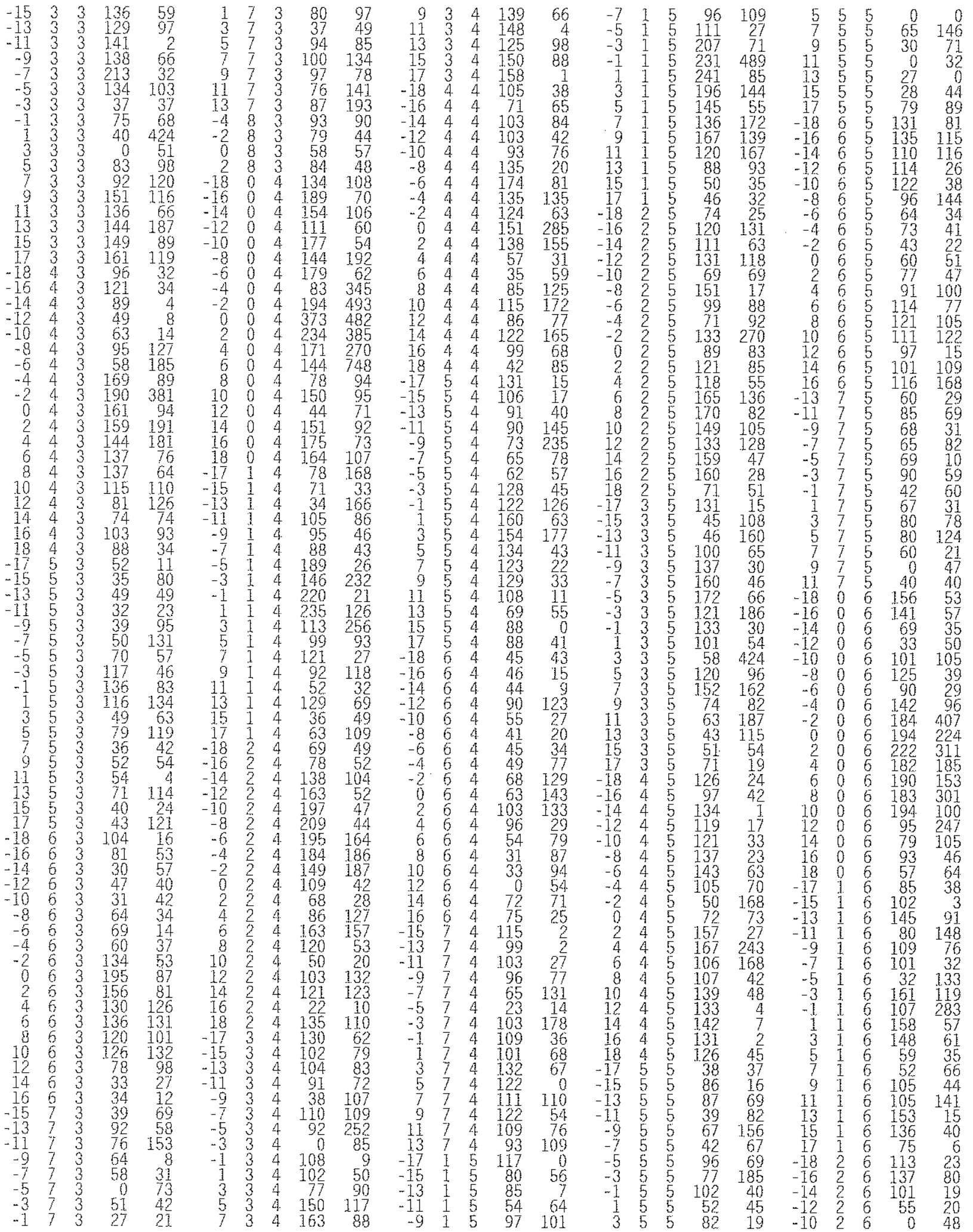


Table observed and calculated structure factors for triazene

Page 3

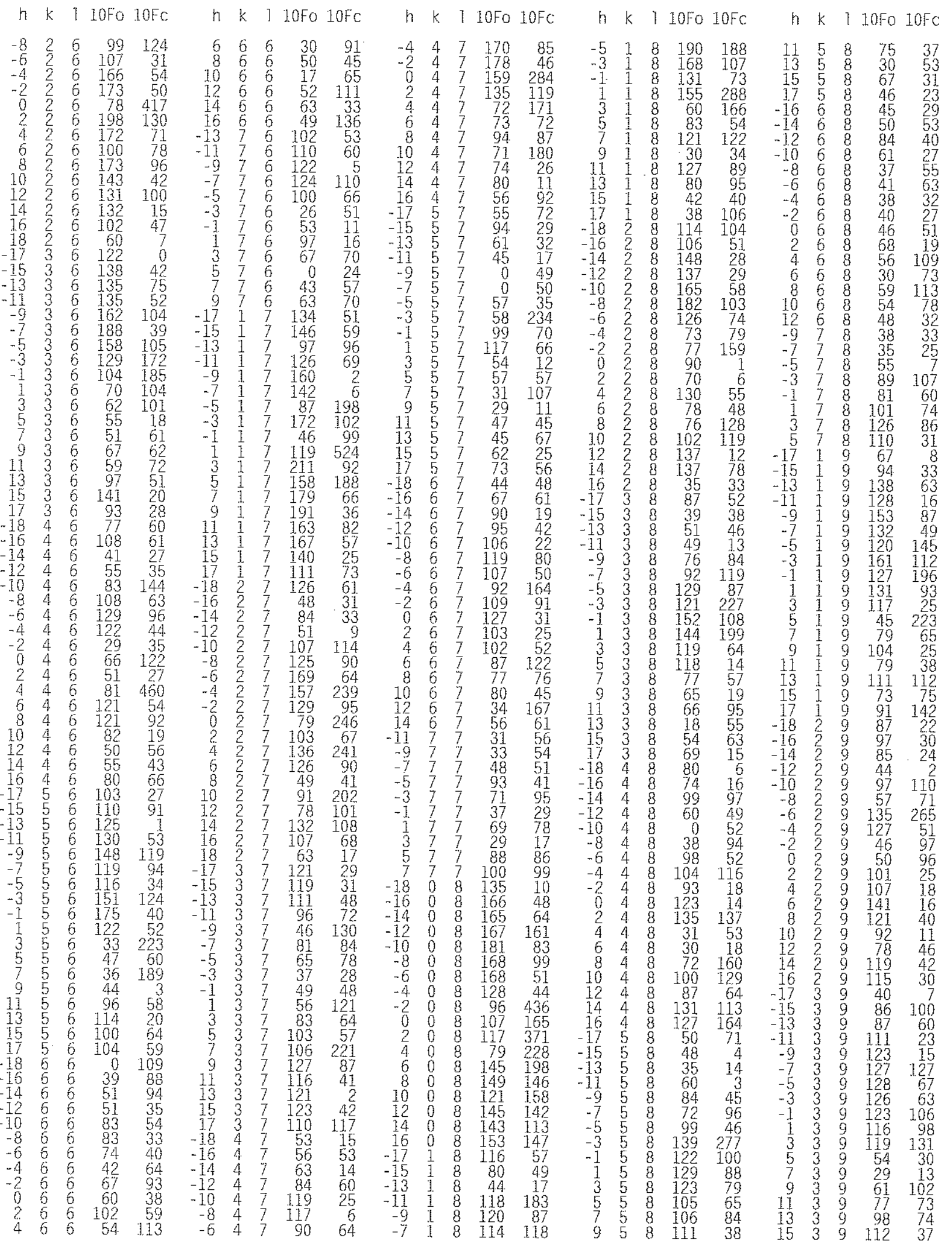


Table . Observed and calculated structure factors for triazene

Page 4

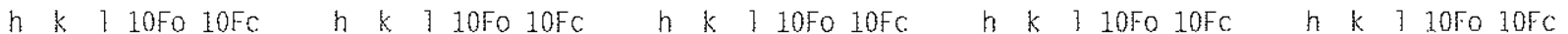

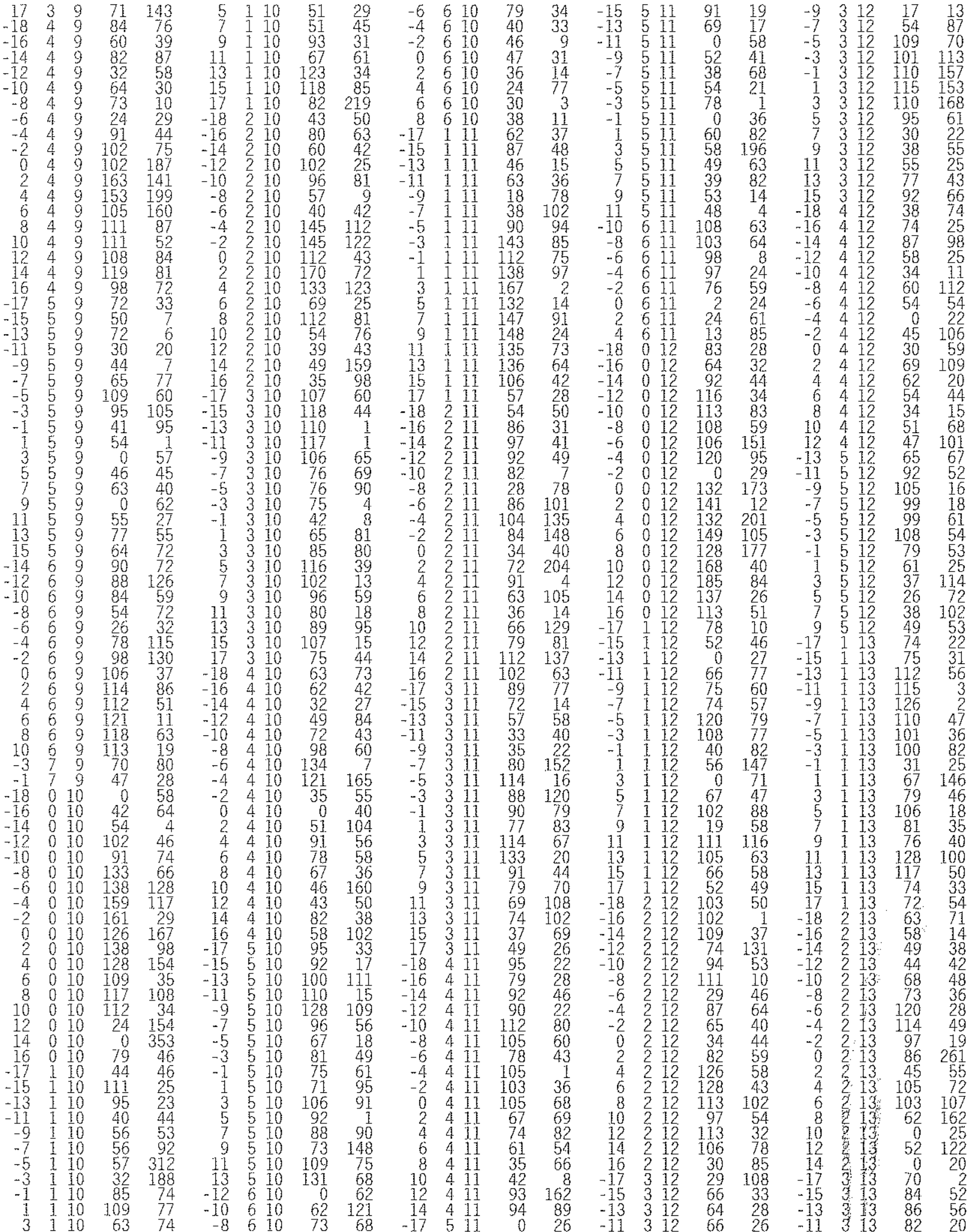




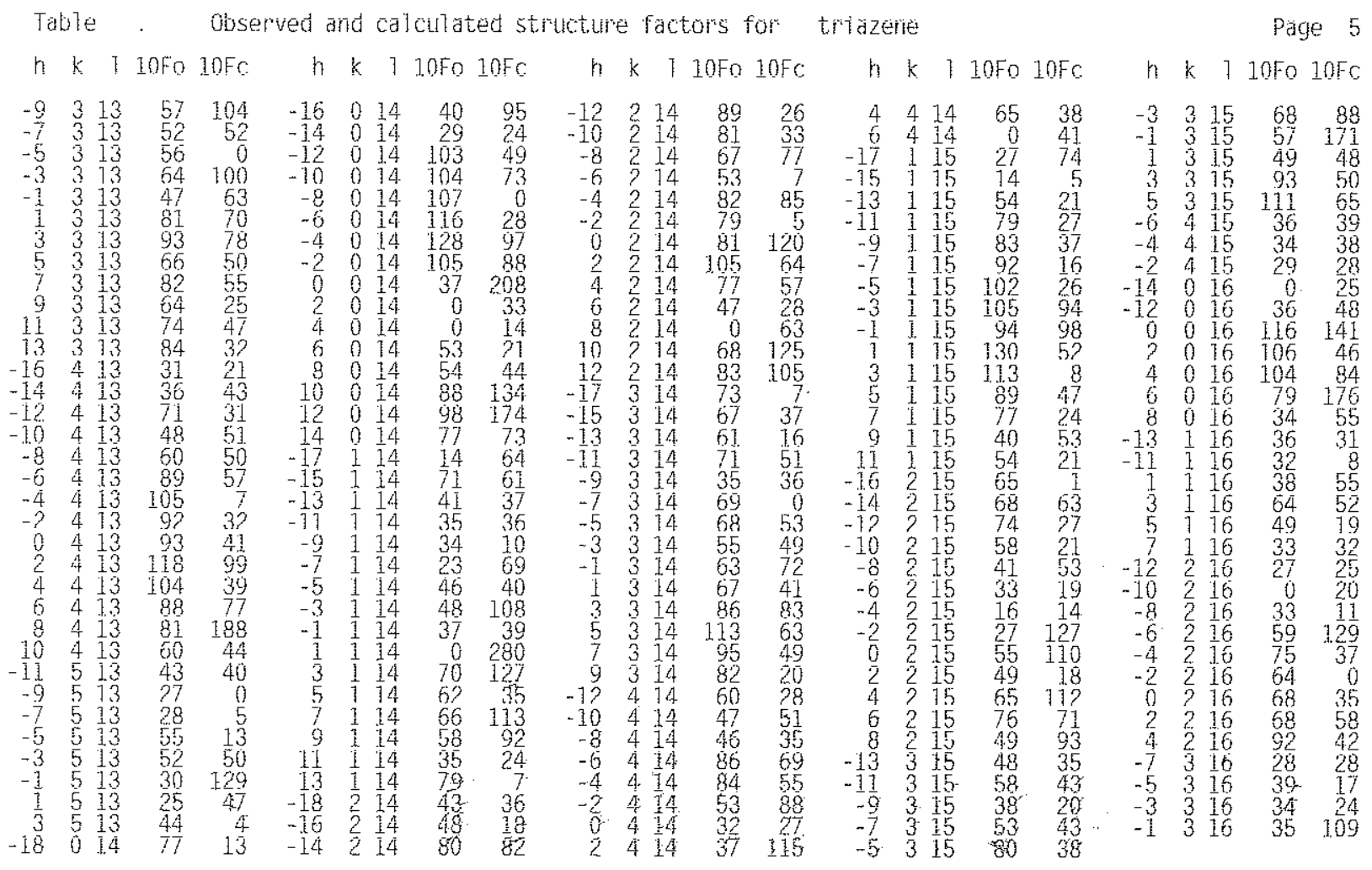

